\title{
On the General Dedekind Sums and Two-Term Exponential Sums
}

\author{
Junli Zhang ${ }^{1}$ and Wenpeng Zhang ${ }^{2}$ \\ ${ }^{1}$ Xian Eurasia University, Xi'an 710065, China \\ ${ }^{2}$ School of Mathematics, Northwest University, Xian 710127, China \\ Correspondence should be addressed to Wenpeng Zhang; wpzhang@nwu.edu.cn \\ Received 5 June 2014; Accepted 20 August 2014; Published 14 October 2014 \\ Academic Editor: Gustaaf Schoukens
}

Copyright (c) 2014 J. Zhang and W. Zhang. This is an open access article distributed under the Creative Commons Attribution License, which permits unrestricted use, distribution, and reproduction in any medium, provided the original work is properly cited.

We use the analytic methods and the properties of Gauss sums to study the computational problem of one kind hybrid mean value involving the general Dedekind sums and the two-term exponential sums, and give an interesting computational formula for it.

\section{Introduction}

Let $q$ be a natural number and $h$ an integer prime to $q$. The classical Dedekind sums

$$
S(h, q)=\sum_{a=1}^{q}\left(\left(\frac{a}{q}\right)\right)\left(\left(\frac{a h}{q}\right)\right),
$$

where

$$
((x))= \begin{cases}x-[x]-\frac{1}{2}, & \text { if } x \text { is not an integer; } \\ 0, & \text { if } x \text { is an integer, }\end{cases}
$$

describes the behaviour of the logarithm of the eta-function (see $[1,2])$ under modular transformations. About the various arithmetical properties of $S(h, q)$, many people had studied it and obtained a series of interesting results; see [3-9].

For example, Wang and Zhang [6] and Wang and Pan [7] had studied the hybrid mean value involving Dedekind sums and two-term exponential sums and proved the computational formulae

$$
\begin{gathered}
\sum_{m=1}^{p-1} \sum_{n=1}^{p-1}|C(m, n, 3,1 ; p)|^{2} \cdot S(m \bar{n}, p) \\
= \begin{cases}p \cdot h_{p}^{2}, & \text { if } p=12 k+7, \\
3 p \cdot h_{p}^{2}, & \text { if } p=12 k+11, \\
0, & \text { if } p=4 k+1,\end{cases}
\end{gathered}
$$

$$
\begin{aligned}
& \sum_{m=1}^{p-1} \sum_{n=1}^{p-1}|C(m, n, 4,2 ; p)|^{2} \cdot S(m \bar{n}, p) \\
& = \begin{cases}2 p \cdot h_{p}^{2}, & \text { if } p=8 k+7, \\
0, & \text { if } p=4 k+1 \text { or } 8 k+3,\end{cases}
\end{aligned}
$$

where $h_{p}$ denotes the class number of the quadratic field $\mathbf{Q}(\sqrt{-p}), \bar{n}$ denotes the solution of the congruence equation $n x \equiv 1 \bmod p$, and the two-term exponential sums $C(m, n, h, k ; q)$ are defined as

$$
C(m, n, h, k ; q)=\sum_{a=1}^{q} e\left(\frac{m a^{h}+n a^{k}}{q}\right),
$$

$e(y)=e^{2 \pi i y}$. Some results related to $C(m, n, h, k ; q)$ can be found in $[10,11]$. 
On the other hand, Zhang [12] introduced a generalized Dedekind sums as follows:

$$
S(h, n ; q)=\sum_{a=1}^{q} \bar{B}_{n}\left(\frac{a}{q}\right) \bar{B}_{n}\left(\frac{a h}{q}\right),
$$

where

$$
\bar{B}_{n}(x)= \begin{cases}B_{n}(x-[x]), & \text { if } x \text { is not an integer } \\ 0, & \text { if } x \text { is an integer, }\end{cases}
$$

$B_{n}(x)$ denotes the $n$th Bernoulli polynomial and $\bar{B}_{n}(x)$ defined for all real $0<x \leq 1$ is called the $n$th Bernoulli periodic function.

If $n=1$, then $S(h, 1 ; q)=S(h, q)$, the classical Dedekind sums. About the arithmetical properties of $S(h, n ; q)$ and $B_{n}(x)$, one can find them in $[3,12]$. In this paper as a note of $[6,7]$, we consider the following hybrid mean value:

$$
\sum_{u=1}^{p-1} \sum_{v=1}^{p-1} C(m, u, h, k ; p) \cdot C(m, v, h, k ; p) \cdot S(u \cdot \bar{v}, n ; p)
$$

and use the analytic methods and the properties of Gauss sums to give an exact computational formula for (7). That is, we will prove the following conclusion.

Theorem 1. Let $p \geq 3$ be a prime and $n$ any positive integer. Then for any positive integers $h$ and $k$ with $(h k, p-1)=1$ and integer $m$ with $(m, p)=1$, one has the identity

$$
\begin{aligned}
& \sum_{u=1}^{p-1} \sum_{v=1}^{p-1} C(u, m, h, k ; p) \cdot C(v, m, h, k ; p) \cdot S(u \cdot \bar{v}, n ; p) \\
& =p^{2} \cdot S(1, n ; p) .
\end{aligned}
$$

For $n=1,2$, and 3, from our theorem we may immediately deduce the following.

Corollary 2. Let $p \geq 3$ be a prime. Then for any positive integers $h$ and $k$ with $(h k, p-1)=1$ and integer $m$ with $(m, p)=1$, one has the identity

$$
\begin{aligned}
& \sum_{u=1}^{p-1} \sum_{v=1}^{p-1} C(u, m, h, k ; p) \cdot C(v, m, h, k ; p) \cdot S(u \cdot \bar{v}, 1 ; p) \\
& \quad=\frac{1}{12} \cdot p(p-1)(p-2) .
\end{aligned}
$$

Corollary 3. Let $p \geq 3$ be a prime. Then for any positive integers $h$ and $k$ with $(h k, p-1)=1$ and integer $m$ with $(m, p)=1$, one has the identity

$$
\begin{gathered}
\sum_{u=1}^{p-1} \sum_{v=1}^{p-1} C(u, m, h, k ; p) \cdot C(v, m, h, k ; p) \cdot S(u \cdot \bar{v}, 2 ; p) \\
=\frac{1}{180} \cdot \frac{(p-1)\left(p^{3}-4 p^{2}+6 p+6\right)}{p} .
\end{gathered}
$$

Corollary 4. Let $p \geq 3$ be a prime. Then for any positive integers $h$ and $k$ with $(h k, p-1)=1$ and integer $m$ with $(m, p)=1$, one has the identity

$$
\begin{array}{r}
\sum_{u=1}^{p-1} \sum_{v=1}^{p-1} C(u, m, h, k ; p) \cdot C(v, m, h, k ; p) \cdot S(u \cdot \bar{v}, 3 ; p) \\
=\frac{1}{840} \cdot \frac{(p-2)(p-1)(p+1)(p+2)\left(p^{2}+5\right)}{p^{3}} .
\end{array}
$$

For general integer $q>3$, whether there exists an exact computational formula for the hybrid mean value

$$
\sum_{u=1}^{q} \sum_{v=1}^{q} C(u, m, h, k ; q) \cdot C(v, m, h, k ; q) \cdot S(u \cdot \bar{v}, n ; q)
$$

is an open problem, where $h$ and $k$ are positive integers with $(k h, \phi(q))=1$ and $(m, q)=1$.

\section{Several Lemmas}

In this section, we will give two lemmas, which are necessary in the proof of our theorem. Hereinafter, we will use many properties of character sums and Gauss sums; all of these can be found in [13], so they will not be repeated here. First we have the following.

Lemma 1. Let $p$ be an odd prime and $\chi$ any nonprincipal character $\bmod p$. Then for any positive integers $h$ and $k \geq 1$ with $(h k, p-1)=1$ and any integer $m$, one has the identity

$$
\sum_{u=1}^{p-1} \chi(u) \cdot C(u, m, h, k ; p)=\chi^{\bar{k} \cdot h}(m) \cdot \tau(\chi) \cdot \tau\left(\bar{\chi}^{\bar{k} \cdot h}\right),
$$

where $\tau(\chi)=\sum_{a=1}^{p-1} \chi(a) e(a / p)$ denotes the Gauss sums.

Proof. From the definitions of $C(u, m, h, k ; p)$ and Gauss sums we have

$$
\begin{aligned}
\sum_{u=1}^{p-1} \chi & (u) \cdot C(u, m, h, k ; p) \\
& =\sum_{u=1}^{p-1} \chi(u) \sum_{a=1}^{p} e\left(\frac{u a^{h}+m a^{k}}{p}\right) \\
& =\sum_{a=1}^{p} \sum_{u=1}^{p-1} \chi(u) \cdot e\left(\frac{u a^{h}+m a^{k}}{p}\right) \\
& =\tau(\chi) \sum_{a=1}^{p-1} \bar{\chi}^{h}(a) \cdot e\left(\frac{m a^{k}}{p}\right) .
\end{aligned}
$$

Since $(h k, p-1)=1$, then there exits one integer $\bar{k}$ such that $\bar{k} \cdot k \equiv 1 \bmod (p-1)$ and $(\bar{k}, p-1)=1$. From the properties of reduced residue system mod $p$ we know that if $a$ pass through a reduced residue system $\bmod p$, then $a^{\bar{k}}$ also 
pass through a reduced residue system $\bmod p$. So from (14) and Fermat little theorem we have

$$
\begin{aligned}
\sum_{u=1}^{p-1} \chi(u) \cdot C(u, m, h, k ; p) & \\
= & \tau(\chi) \sum_{a=1}^{p-1} \bar{\chi}^{h}\left(a^{\bar{k}}\right) \cdot e\left(\frac{m a^{\bar{k} \cdot k}}{p}\right) \\
= & \tau(\chi) \sum_{a=1}^{p-1} \bar{\chi}^{\bar{k} \cdot h}(a) \cdot e\left(\frac{m a}{p}\right) \\
= & \chi^{\bar{k} \cdot h}(m) \cdot \tau(\chi) \cdot \tau\left(\bar{\chi}^{\bar{k} \cdot h}\right) .
\end{aligned}
$$

This proves Lemma 1.

Lemma 2. Let $q \geq 3$ be an integer and $h$ any integer with $(h, q)=1$. Then for any positive integer $n$, one has the following identities.

(i) If $n$ is an odd number, then

$$
S(h, n ; q)=\frac{(n !)^{2}}{4^{n-1} q^{2 n-1} \pi^{2 n}} \sum_{d \mid q} \frac{d^{2 n}}{\phi(d)} \sum_{\substack{\chi \bmod d \\ \chi(-1)=-1}} \chi(h)|L(n, \chi)|^{2} .
$$

(ii) If $n$ is an even number, then

$$
\begin{aligned}
S(h, n ; q)= & \frac{(n !)^{2}}{4^{n-1} q^{2 n-1} \pi^{2 n}} \sum_{d \mid q} \frac{d^{2 n}}{\phi(d)} \\
& \times \sum_{\substack{\chi \bmod d \\
\chi(-1)=1}} \chi(h)|L(n, \chi)|^{2}-\frac{(n !)^{2}}{4^{n-1} \pi^{2 n}} \cdot \zeta^{2}(n),
\end{aligned}
$$

where $L(1, \chi)$ denotes the Dirichlet L-function corresponding to character $\chi \bmod d$ and $\zeta(s)$ is the famous Riemann zetafunction.

Proof. See [12].

\section{Proof of the Theorem}

In this section, we will complete the proof of our theorem. If $n$ is an odd number, then note that for any nonprincipal character $\chi \bmod p,|\tau(\chi)|=\sqrt{p}$ and $\tau(\chi) \cdot \tau(\bar{\chi})=$ $\bar{\chi}(-1) \tau(\chi) \overline{\tau(\chi)}=\bar{\chi}(-1) \cdot p$. So for any integer $u$ with $(u, p)=$ 1 , from (i) of Lemma 2 we have

$$
\begin{gathered}
\sum_{u=1}^{p-1} \sum_{v=1}^{p-1} C(u, m, h, k ; p) \cdot C(v, m, h, k ; p) \cdot S(u \cdot \bar{v}, n ; p) \\
=\frac{(n !)^{2} \cdot p}{4^{n-1}(p-1) \pi^{2 n}}
\end{gathered}
$$

$$
\begin{aligned}
& \times \sum_{\substack{\chi \bmod \\
\chi(-1)=-1}} \sum_{\substack{p=1 \\
\chi-1}}^{p-1} C(u, m, h, k ; p) \\
& \text { - } C(v, m, h, k ; p) \chi(u \cdot \bar{v})|L(n, \chi)|^{2} \\
& =\frac{(n !)^{2} \cdot p}{4^{n-1}(p-1) \pi^{2 n}} \sum_{\substack{\chi \bmod p \\
\chi(-1)=-1}} \bar{\chi}(-1) \chi^{\bar{k} \cdot h}(-1) \cdot p^{2} \\
& \cdot|L(n, \chi)|^{2} \\
& =\frac{(n !)^{2} \cdot p^{3}}{4^{n-1}(p-1) \pi^{2 n}} \sum_{\substack{\chi \bmod p \\
\chi(-1)=-1}}|L(n, \chi)|^{2}=p^{2} \cdot S(1, n ; p) .
\end{aligned}
$$

If $n \geq 2$ is an even number, then note that $(k, p-1)=1$ and the identity

$$
\begin{aligned}
\sum_{u=1}^{p-1} C & (u, m, h, k ; p) \\
& =\sum_{a=1}^{p} \sum_{u=1}^{p-1} e\left(\frac{u a^{h}+m a^{k}}{p}\right) \\
& =p-1+\sum_{a=1}^{p-1} e\left(\frac{m a^{k}}{p}\right) \sum_{u=1}^{p-1} e\left(\frac{u a^{h}}{p}\right) \\
& =p-1-\sum_{a=1}^{p-1} e\left(\frac{m a}{p}\right)=p .
\end{aligned}
$$

From (ii) of Lemma 2 and the method of proving (18) we have

$$
\begin{aligned}
\sum_{u=1}^{p-1} \sum_{v=1}^{p-1} C & (u, m, h, k ; p) \cdot C(v, m, h, k ; p) \cdot S(u \cdot \bar{v}, n ; p) \\
= & \frac{(n !)^{2} \cdot p}{4^{n-1}(p-1) \pi^{2 n}} \\
& \times \sum_{\chi \bmod p} \sum_{u=1}^{p-1} \sum_{v=1}^{p-1} C(u, m, h, k ; p) \\
& \quad \chi(-1)=1 \\
& +\frac{\left(n ! \chi_{0}\right.}{4^{n-1} p^{2 n-1} \pi^{2 n}} \cdot \zeta^{2}(n)+\frac{(n !)^{2} \cdot p}{4^{n-1}(p-1) \pi^{2 n}} \\
& \cdot p^{2} \cdot\left|L\left(n, \chi_{0}\right)\right|^{2}-\frac{(n !)^{2} \cdot p^{2}}{4^{n-1} \pi^{2 n}} \zeta^{2}(n)
\end{aligned}
$$




$$
\begin{aligned}
= & \frac{(n !)^{2} \cdot p^{3}}{4^{n-1}(p-1) \pi^{2 n}} \sum_{\substack{\chi \bmod _{p} \\
\chi(-1)=1}}|L(n, \chi)|^{2} \\
& +\frac{(n !)^{2} \cdot p^{2}}{4^{n-1} p^{2 n-1} \pi^{2 n}} \cdot \zeta^{2}(n)-\frac{(n !)^{2} \cdot p^{2}}{4^{n-1} \pi^{2 n}} \zeta^{2}(n) \\
= & \frac{(n !)^{2} \cdot p^{2}}{4^{n-1} p^{2 n-1} \pi^{2 n}} \sum_{d \mid p} \frac{d^{2 n}}{\phi(d)} \sum_{\chi \bmod d}|L(n, \chi)|^{2} \\
& -\frac{(n !)^{2} \cdot p^{2}}{4^{n-1} \pi^{2 n}} \cdot \zeta^{2}(n) \\
= & p^{2} \cdot S(1, n ; p) .
\end{aligned}
$$

Combining (18) and (20) we may immediately complete the proof of our theorem.

From the definition of $S(1,1 ; p)$ we have

$$
\begin{aligned}
S(1,1 ; p) & =\sum_{a=1}^{p-1}\left(\frac{a}{p}-\frac{1}{2}\right)^{2}=\frac{1}{12}\left(p-3+\frac{2}{p}\right) \\
& =\frac{(p-1)(p-2)}{12 p} .
\end{aligned}
$$

Combining (18) and (21) we can deduce the identity

$$
\begin{gathered}
\sum_{u=1}^{p-1} \sum_{v=1}^{p-1} C(u, m, h, k ; p) \cdot C(v, m, h, k ; p) \cdot S(u \cdot \bar{v}, 1 ; p) \\
=\frac{1}{12} \cdot p(p-1)(p-2) .
\end{gathered}
$$

This proves Corollary 2.

If $n=2$, then note that $B_{2}(x)=x^{2}-x+1 / 6$; we have

$$
\begin{aligned}
S(1,2 ; q) & =\sum_{a=1}^{q-1} \bar{B}_{2}^{2}\left(\frac{a}{q}\right)=\sum_{a=1}^{q-1}\left(\frac{a^{2}}{q^{2}}-\frac{a}{q}+\frac{1}{6}\right)^{2} \\
& =\frac{q}{180}-\frac{1}{36}+\frac{1}{18 q}-\frac{1}{30 q^{3}} .
\end{aligned}
$$

From (20) and (23) we have the identity

$$
\begin{gathered}
\sum_{u=1}^{p-1} \sum_{v=1}^{p-1} C(u, m, h, k ; p) \cdot C(v, m, h, k ; p) \cdot S(u \cdot \bar{v}, 2 ; p) \\
=\frac{1}{180} \cdot \frac{(p-1)\left(p^{3}-4 p^{2}+6 p+6\right)}{p} .
\end{gathered}
$$

This proves Corollary 3.

$$
\begin{aligned}
S(1,3 ; q) & =\sum_{a=1}^{q-1} \bar{B}_{3}^{2}\left(\frac{a}{q}\right)=\sum_{a=1}^{q-1}\left(\frac{a^{3}}{q^{3}}-\frac{3}{2} \frac{a^{2}}{q^{2}}+\frac{1}{2} \frac{a}{q}\right)^{2} \\
& =\frac{q}{840}-\frac{1}{40 q^{3}}+\frac{1}{42 q^{5}} .
\end{aligned}
$$

From (18) and (23) we have the identity

$$
\begin{array}{r}
\sum_{u=1}^{p-1} \sum_{v=1}^{p-1} C(u, m, h, k ; p) \cdot C(v, m, h, k ; p) \cdot S(u \cdot \bar{v}, 3 ; p) \\
=\frac{1}{840} \cdot \frac{(p-2)(p-1)(p+1)(p+2)\left(p^{2}+5\right)}{p^{3}} .
\end{array}
$$

This completes the proof of Corollary 4.

\section{Conflict of Interests}

The authors declare that there is no conflict of interests regarding the publication of this paper.

\section{Acknowledgments}

The authors would like to thank the referee for his very helpful and detailed comments, which have significantly improved the presentation of this paper. This work is supported by the P. S. F. (2013JZ001) and N.S.F. (11371291) of China.

\section{References}

[1] H. Rademacher, "On the transformation of $\log \eta(\tau)$, , The Journal of the Indian Mathematical Society, vol. 19, pp. 25-30, 1955.

[2] H. Rademacher and E. Grosswald, Dedekind Sums, Carus Mathematical Monographs, The Mathematical Association of America, Washington, DC, USA, 1972.

[3] T. M. Apostol, Modular Functions and Dirichlet Series in Number Theory, Springer, New York, NY, USA, 1976.

[4] C. Jia, "On the mean value of Dedekind sums," Journal of Number Theory, vol. 87, no. 2, pp. 173-188, 2001.

[5] J. B. Conrey, E. Fransen, R. Klein, and C. Scott, "Mean values of Dedekind sums," Journal of Number Theory, vol. 56, no. 2, pp. 214-226, 1996.

[6] T. Wang and W. Zhang, "On hybrid mean value of Dedekind sums and two-term exponential sums," Frontiers of Mathematics in China, vol. 6, no. 3, pp. 557-563, 2011.

[7] T. Wang and X. Pan, "The mean value involving Dedekind sums and two-term exponential sums," Science China: Mathematics, vol. 55, no. 3, pp. 557-565, 2012.

[8] W. Zhang, "On the mean values of Dedekind sums," Journal de Théorie des Nombres de Bordeaux, vol. 8, no. 2, pp. 429-442, 1996.

[9] W. Zhang, "A note on the mean square value of the Dedekind sums," Acta Mathematica Hungarica, vol. 86, no. 4, pp. 275-289, 2000.

[10] T. Cochrane and Z. Zheng, "Upper bounds on a two-term exponential sum," Science in China A, vol. 44, no. 8, pp. 10031015, 2001.

[11] T. Cochrane, J. Coffelt, and C. Pinner, "A further refinement of Mordell's bound on exponential sums," Acta Arithmetica, vol. 116, no. 1, pp. 35-41, 2005.

[12] W. P. Zhang, "On the general Dedekind sums and one kind identities of Dirichlet L-functions," Acta Mathematica Sinica, vol. 44, no. 2, pp. 269-272, 2001.

[13] T. M. Apostol, Introduction to Analytic Number Theory, Springer, New York, NY, USA, 1976. 


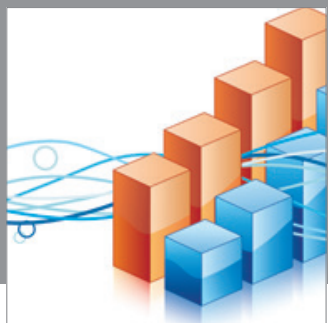

Advances in

Operations Research

mansans

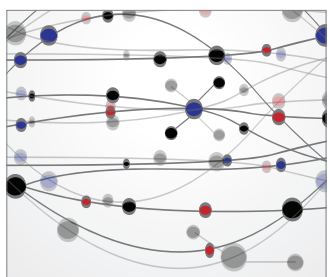

The Scientific World Journal
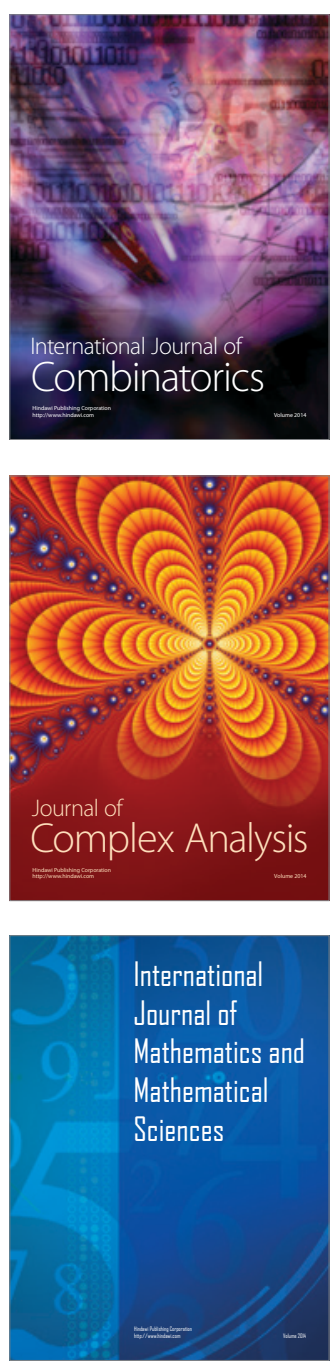
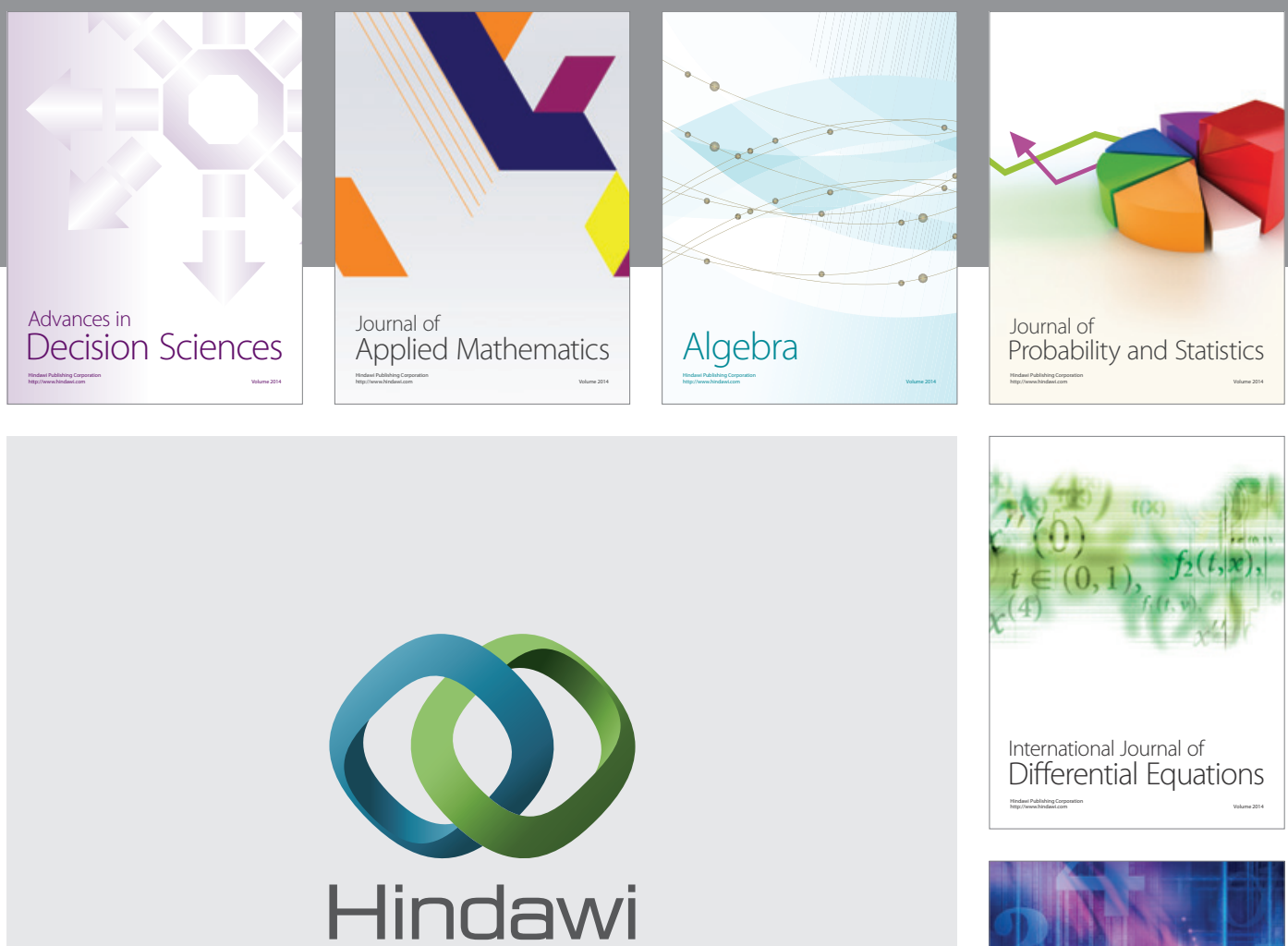

Submit your manuscripts at http://www.hindawi.com
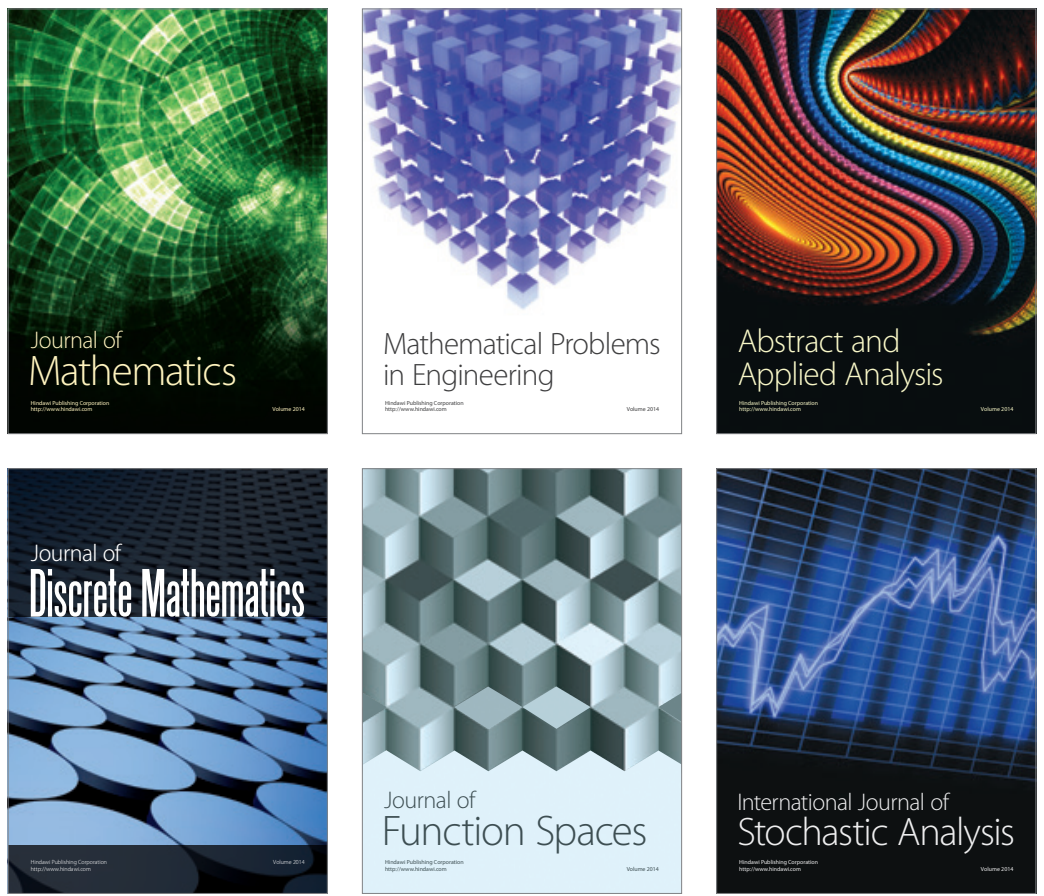

Journal of

Function Spaces

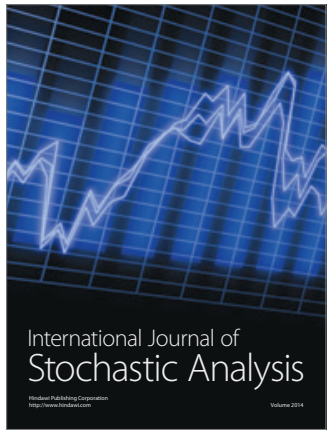

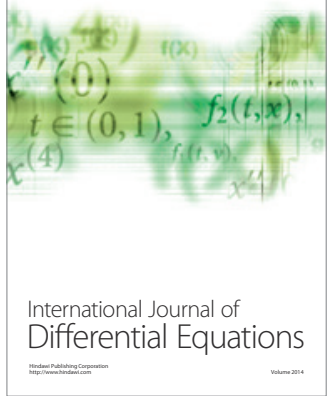
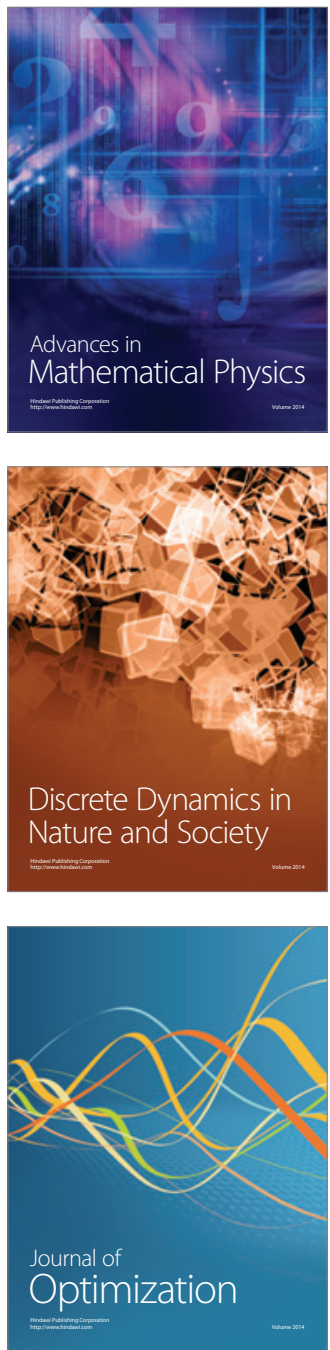